\title{
Acknowledgment to Reviewers of Journal of Sensor and Actuator Networks in 2020
}

\author{
Journal of Sensor and Actuator Networks Editorial Office
}

Citation: Journal of Sensor and Actuator Networks Editorial Office.

Acknowledgment to Reviewers of Journal of Sensor and Actuator

Networks in 2020. J. Sens. Actuator Netw. 2021, 10, 4. https://doi.org/ 10.3390/jsan10010004

Published: 16 January 2021

Publisher's Note: MDPI stays neutral with regard to jurisdictional claims in published maps and institutional affiliations.

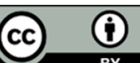

Copyright: $(2021$ by the authors. Licensee MDPI, Basel, Switzerland. This article is an open access article distributed under the terms and conditions of the Creative Commons Attribution (CC BY) license (http://creativecommons.org/licenses/by/4.0/).
MDPI AG, St. Alban-Anlage 66, 4052 Basel, Switzerland

Peer review is the driving force of journal development, and reviewers are gatekeepers who ensure that Journal of Sensor and Actuator Networks maintains its standards for the high quality of its published papers. Thanks to the cooperation of our reviewers, in 2020, the median time to first decision was 21 days and the median time to publication was 55 days. The editors would like to express their sincere gratitude to the following reviewers for their precious time and dedication, regardless of whether the papers were finally published:

\begin{tabular}{|c|c|}
\hline Abdullah, Oday Ibraheem & Crotti, Gianluca \\
\hline Abenza, Pedro Pablo Garrido & D'Alvia, Livio \\
\hline Achir, Nadjib & Dai, Xuewu \\
\hline Ahamed, Tofael & De La Llana Calvo, Álvaro \\
\hline Ahmad, Tanveer & Dennis, Louise \\
\hline Aktan, A. Emin & Di Maio, Antonio \\
\hline Albano, Michele & Duarte Correia, Sérgio \\
\hline Albuquerque, Victor & Dwivedi, Ashutosh Dhar \\
\hline Alcarria, Ramón & Estrada-López, Johan J. \\
\hline Alsafrjalani, Hammam & Fabian, Ralf \\
\hline Amayri, Manar & Fedele, Rosario \\
\hline Anisi, Mohammad & Flatau, Alison B. \\
\hline Anochi, Juliana & Fojcik, Marcin \\
\hline Araujo, Alvaro & $\mathrm{Fu}$, Xiuwen \\
\hline Arcia-Moret, Andrés & Fuentes-Pérez, Juan Francisco \\
\hline Bahsi, Hayretdin & Furtado, António \\
\hline Beben, Damian & Gasulla, Manuel \\
\hline Bhargava, Kriti & Ghoreyshi, Seyed Mohammad \\
\hline Biancolillo, Alessandra & Giannoulis, Spilios \\
\hline Bol, David & González-Briones, Alfonso \\
\hline Boragno, Corrado & Grazia, Carlo Augusto \\
\hline Bradbury, Matthew & Grega, Wojciech \\
\hline Buades Rubio, Jose Maria & Guido, Matrella \\
\hline Bures, Miroslav & Haller, Piroska \\
\hline Campagnaro, Filippo & Han, Ruisong \\
\hline Castellanos Garzón, José Antonio & He, Yihai \\
\hline Castillejo Parrilla, Pedro & He, Yunze \\
\hline Cavenaghi, Marcos & Henriques, Felipe Da Rocha \\
\hline Cerro, Gianni & Holubek, Radovan \\
\hline Chai, Kok Keong & Hornillo Mellado, Susana \\
\hline Chehri, Abdellah & $\mathrm{Hu}$, Xiaoping \\
\hline Chen, Liang-Bi & Huang, Chung-Jen \\
\hline Chen, Lien-Wu & Hussein, Moustafa \\
\hline Chi, Haoran & Hwang, Wen-Jyi \\
\hline Choi, Wooyeol & Ikerionwu, Charles \\
\hline Cornetta, Gianluca & Ishihara, Susumu \\
\hline
\end{tabular}


Jang, Jongwook

Jang, Yeong

Jesus, Goncalo

Kabassi, Katerina

Kadel, Rajan

Kaiwartya, Omprakash

Kandris, Dionisis

Kapoulas, Vaggelis

Katsaros, Konstantinos

Keoh, Sye Loong

Khan, Tareq

Kim, Daehee

Kim, Jeehyeong

Kim, Moonseong

Kong, Kian Hau

Kontoni, Denise-Penelope N.

Kostrzewski, Mariusz

Koudouridis, Georgios P.

Kristian, Yosi

Kuok, Sin-Chi

Kwak, Moon K.

Lavric, Alexandru

Lee, Il-Gu

Lee, Jong Yong

Lee, Kyu-haeng

Lee, Tian-Fu

Leng, Jiewu

Leonardi, Luca

Li, Chengqing

Liang, Chiu-kuo

Lim, Sangkyu

Lin, Gong-Ru

Lin, Tsung-Hung

Liu, Anfeng

Liu, Jing

Liu, Pei

Liu, Shijun

Liu, Shuaiqi

Liu, Zhuofu

Llaria, Alvaro

Lloret, Jaime

Lo, Shi-Wei

Lukas, Vojtěch

Machaj, Juraj

Maennel, Olaf Manuel

Marchiori, Alan

Marrero, Domingo

MARS, Kamel

Martin-Fernandez, Cristian

Mascardi, Viviana

Masini, Barbara Mavi

Mata Miquel, Christian

Mazurkiewicz, Dariusz

Memmolo, Vittorio

Men, Zhongxian

Meuser, Tobias

Mihailescu, Marius Iulian
Moh, Sangman

Monteleone, Salvatore

Moreno-Eguilaz, Juan Manuel

Moreno-Moreno, Carlos D.

Morozs, Nils

Moscholios, Ioannis D.

Muheidat, Fadi

Mukai, Hiroaki

Nguyen, Linh

Nguyen, Tien-Dung

Nkenyereye, Lewis

Nóbrega, Luís

Novikova, Evgenia

Obreja, Serban Georgica

Orúe López, Amalia Beatriz

Pasolini, Gianni

Pau, Giovanni

Peleato, Borja

Pescaru, Dan

Piltan, Farzin

Pirinen, Pekka

Pletersek, Anton

Poniszewska-Maranda, Aneta

Potamitis, Ilyas

Pozzebon, Alessandro

$\mathrm{Pu}$, Cong

Qamar, Faizan

Rahman, Juber

Refaey, Ahmed

Reisenfeld, Sam

Rim, Minjoong

Rizal, Conrad

Roussey, Catherine

Sánchez, Bordel

Saxena, Sahaj

See, Chan H.

Senatore, Gennaro

Shah, Purav

Shah, Syed Tariq

Shahrour, Isam

Shanmugam, Bharanidharan

Shibata, Naoki

Shiryayev, Oleg

Sisinni, Emiliano

Sobue, Shinichi

Socas, Rafael

Soliman, Hamdy

Song, Kang

Song, Shuang

Soylu, Ahmet

Stencel, Marek

Stepien, Jacek

Stetter, Ralf

Strle, Drago

$\mathrm{Su}$, Ruoyu

Sun, Hua

Sun, Yan 
Takabayashi, Kento

Tardif, Pierre Martin

Tari, Zahir

Tashakori, Shervin

Thakur, Subhasis

Thurow, Kerstin

Tomarchio, Orazio

Trakadas, Panagiotis

Tsiropoulou, Eirini Eleni

Tsukada, Manabu

Valente, Antonio

Vangelista, Lorenzo

Varga, Pal

Vasa, Rajesh

Vasilateanu, Andrei

Vergaz, Ricardo

Vincke, Bastien

Vinel, Alexey

Vinueza Naranjo, Paola Gabriela

Violettas, George E.

Wang, Hongxia
Wang, Kai

Węglarski, Mariusz

Weitzen, Jay A.

Wen, Chih-Yu

Wojciechowski, Tomasz

$\mathrm{Wu}$, Jianfeng

Xiang, Jiawei

Xiong, Wen

$\mathrm{Xu}, \mathrm{Chi}$

Xue, Zhidong

Yang, Mau-Tsuen

Zabini, Flavio

Zefferer, Thomas

Zhang, Su

Zhang, Xian-Ming

Zhao, Jinling

Zhao, Juan

Zhou, Nan

Zhuang, Yanyan

Zielinski, Zbigniew 